\title{
The extent of carbapenemase-encoding genes in public genome sequences
}

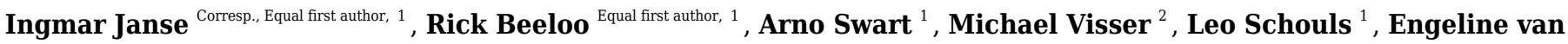 \\ Duijkeren ${ }^{1}$, Mark W. J. van Passel ${ }^{1,3}$ \\ ${ }^{1}$ Center for Infectious Disease Control, National Institute for Public Health and the Environment, Bilthoven, Utrecht, the Netherlands \\ 2 Sequencing and Bioinformatics, Netherlands Food and Consumer Product Safety Authority (NVWA), Utrecht, Utrecht, the Netherlands \\ 3 Ministry of Health, Welfare and Sport, The Hague, the Netherlands \\ Corresponding Author: Ingmar Janse \\ Email address: ingmar.janse@rivm.nl
}

Genome sequences provide information on the genetic elements present in an organism, and currently there are databases containing hundreds of thousands of bacterial genome sequences. These repositories allow for mining patterns concerning antibiotic resistance gene occurrence in both pathogenic and non-pathogenic bacteria in e.g. natural or animal environments, and link these to relevant metadata such as bacterial host species, year and country of isolation, and co-occurrence with other resistance genes. In addition, the advances in the prediction of mobile genetic elements, and discerning chromosomal from plasmid DNA, broadens our view on the mechanism mediating dissemination. In this study we utilize the vast amount of data in the public database PATRIC to investigate the dissemination of carbapenemase-encoding genes (CEGs), the emergence and spread of which is considered a grave public health concern. Based on publicly available genome sequences from PATRIC and manually curated CEG sequences from the beta lactam database, we found 7,964 bacterial genomes, belonging to at least 70 distinct species, that carry in total 9,892 CEGs, amongst which bla ${ }_{\mathrm{NDM}}, b / a_{\mathrm{OXA}}, b / a_{\mathrm{VIM}}, b / a_{\mathrm{IMP}}$ and b/a $a_{\mathrm{KPC}}$. We were able to distinguish between chromosomally located resistance genes $(4,137 ; 42 \%)$ and plasmid-located resistance genes $(5,753 ; 58 \%)$. We found that a large proportion of the identified CEGs were identical, i.e. displayed $100 \%$ nucleotide similarity in multiple bacterial species (8,361 out of 9,892 genes; $85 \%)$. For example, the New Delhi metallobeta-lactamase NDM-1 was found in 42 distinct bacterial species, and present in seven different environments. Our data show the extent of carbapenem-resistance far beyond the canonical species Acetinobacter baumannii, Klebsiella pneumoniae or Pseudomonas aeruginosa. These types of data complement previous systematic reviews, in which carbapenem-resistant Enterobacteriaceae were found in wildlife, livestock and companion animals. Considering the widespread distribution of CEGs, we see a need for 
comprehensive surveillance and transmission studies covering more host species and environments, akin to previous extensive surveys that focused on extended spectrum beta-lactamases. This may help to fully appreciate the spread of CEGs and improve the understanding of mechanisms underlying transmission, which could lead to interventions minimizing transmission to humans. 


\section{The extent of carbapenemase-encoding genes in} 2 public genome sequences

3

4 Ingmar Janse $^{1 *}$, Rick Beeloo ${ }^{1 *}$, Arno Swart ${ }^{1}$, Michael Visser ${ }^{1}$, Leo Schouls ${ }^{1}$, Engeline van

5 Duijkeren ${ }^{1}$, Mark W. J. van Passel ${ }^{1,2}$

6

$7{ }^{1}$ National Institute for Public Health and the Environment, Center for Infectious Disease Control,

8 Bilthoven, the Netherlands

9 2Netherlands Food and Consumer Product Safety Authority (NVWA), Sequencing and

10 Bioinformatics, Utrecht, the Netherlands

$11{ }^{3}$ Ministry of Health, Welfare and Sport, The Hague, the Netherlands

12

13

14

15

16

17

18

19 *shared first authorship

Corresponding Author:

Ingmar Janse, National Institute for Public Health and the Environment, Center for Infectious

Disease Control, Bilthoven, the Netherlands

Email address: ingmar.janse@,rivm.nl 


\section{Abstract}

21

22

23

24

25

26

27

28

29

30

31

32

33

34

35

36

37

38

39

40

41

42

43

44

45

46

47

48

49

Genome sequences provide information on the genetic elements present in an organism, and currently there are databases containing hundreds of thousands of bacterial genome sequences. These repositories allow for mining patterns concerning antibiotic resistance gene occurrence in both pathogenic and non-pathogenic bacteria in e.g. natural or animal environments, and link these to relevant metadata such as bacterial host species, year and country of isolation, and co-occurrence with other resistance genes. In addition, the advances in the prediction of mobile genetic elements, and discerning chromosomal from plasmid DNA, broadens our view on the mechanism mediating dissemination.

In this study we utilize the vast amount of data in the public database PATRIC to investigate the dissemination of carbapenemase-encoding genes (CEGs), the emergence and spread of which is considered a grave public health concern.

Based on publicly available genome sequences from PATRIC and manually curated CEG sequences from the beta lactam database, we found 7,964 bacterial genomes, belonging to at least 70 distinct species, that carry in total 9,892 CEGs, amongst which bla $a_{\mathrm{NDM}}, b l a_{\mathrm{OXA}}, b l a_{\mathrm{VIM}}, b l a_{\mathrm{IMP}}$ and $b l a_{\mathrm{KPC}}$. We were able to distinguish between chromosomally located resistance genes $(4,137$; $42 \%)$ and plasmid-located resistance genes $(5,753 ; 58 \%)$. We found that a large proportion of the identified CEGs were identical, i.e. displayed $100 \%$ nucleotide similarity in multiple bacterial species $(8,361$ out of 9,892 genes; $85 \%)$. For example, the New Delhi metallo-beta-lactamase NDM-1 was found in 42 distinct bacterial species, and present in seven different environments. Our data show the extent of carbapenem-resistance far beyond the canonical species Acetinobacter baumannii, Klebsiella pneumoniae or Pseudomonas aeruginosa.

These types of data complement previous systematic reviews, in which carbapenem-resistant Enterobacteriaceae were found in wildlife, livestock and companion animals. Considering the widespread distribution of CEGs, we see a need for comprehensive surveillance and transmission studies covering more host species and environments, akin to previous extensive surveys that focused on extended spectrum beta-lactamases. This may help to fully appreciate the spread of CEGs and improve the understanding of mechanisms underlying transmission, which could lead to interventions minimizing transmission to humans.

Peer) reviewing PDF | (2020:06:50324:1:2:NEW 11 Jan 2021) 


\section{Introduction}

51

52

53

54

55

56

57

58

59

60

61

62

63

64

65

66

67

68

69

70

71

72

73

74

75

76

77

78

79
Twenty-five years since the sequencing revolution started we see the availability of hundreds of thousands of bacterial genomes [1], enabling a vast number of comparative analyses leading to e.g. virulence factor prediction [2] or metabolic pathway modelling [3]. The extensive genome resource PATRIC [1] includes detailed information on resistance genes [4], and amongst the many different uses for these accumulated data, they also aim at predicting antimicrobial resistance (AMR) phenotypes in individual genomes. However, the accumulation of all genetic data with their associated metadata on bacterial host species, isolation source, year of isolation, and cooccurrence with other clinical resistance genes, also allows for a global view into the dissemination of resistance genes, for example of carbapenemase-encoding genes (CEGs) in high priority pathogens [5].

Recently, Köck et al. [6] performed a systematic literature review on the occurrence of carbapenem-resistant Enterobacteriaceae (CRE). The authors concluded that the prevalence of CRE in wildlife, livestock and companion animals and directly exposed humans poses public health risks, which was further corroborated by a study on the worsening epidemiological situation in Europe of carbapenemase-producing Enterobacteriaceae [7]. The literature review by Köck et al. was limited to Enterobacteriaceae, and environmental prevalence of CRE had not been included, even though genes encoding most acquired carbapenemases are believed to have transferred from environmental bacteria into species with clinical relevance [8]. We propose that evaluating carbapenemase-encoding genes and their associated metadata from genomic databases may add to the broader picture of the distribution of these genes throughout relevant (non-human) environments, and may highlight transmission between bacterial species via mobile genetic elements. These databases can show whether other bacterial species can represent CEG reservoirs, even when the original researchers of these genomes may not have annotated the carbapenemresistance potential of these species. We aim to tabulate carbapenemase-encoding genes from an extensive genome database, in order to appraise their distribution patterns. Our objective was not a comprehensive coverage of all CEG variants, instead we chose a conservative approach for the annotation of CEGs and used identical sequences to highlight their distribution and mobility. 


\section{Materials \& Methods}

81

82

83

84

85

86

87

88

89

90

91

92

93

94

95

96

97

98

99

100

101

102

103

104

105

106

107

108
All available 175,882 bacterial genomes (August 2018) and their corresponding metadata were downloaded from PATRIC [1]. This metadata included information on the isolated bacteria such as species, taxonomic lineage, isolation source (host organisms, e.g. human, or environment, e.g. forest or wastewater). In addition, the nucleotide (.ffn) gene and protein sequences (.faa) were retrieved from the FTP server (ftp.patricbrc.org). Because genome information is recorded in freetext fields which hampers unambiguous analysis, taxonomic lineages for each genome were reannotated at the species and genus level. Species names were harmonized by automatic annotation as well as manual curation. In order to bin isolation source metadata from all genomes correctly, the various formats describing hosts from which bacteria had been isolated were queried against the NCBI taxonomy database to retrieve taxonomic lineages. Standardized host names allowed assignment to groups such as cattle, chicken, and human. Source data from environmental or food isolates were standardized by querying these against manually curated keywords (Supplemental File 1) to assign each genome to specified environments (Supplemental File 2). In case source metadata was absent, the genome was designated "miscellaneous".

To create a reliable antibiotic resistance gene (ARG) set, protein sequences of all PATRIC ARGs identified upon comparison with CARD (indicated by the "source" column in PATRIC annotation) were subjected to the Resistance Gene Identifier (RGI) [9] and only those detected by the protein homolog model with a perfect or strict cut-off were kept. To identify carbapenemase-encoding genes in this dataset, the protein sequences of 566 manually curated CEGs (Supplemental File 3) were obtained from the Beta-lactamase database (BLDB) [10] as it was compiled in August 2018 and detected in the ARG set using TBLASTN (100\% identity and coverage). Although less stringent settings would have identified more potential CEGs, we chose a conservative approach in order to avoid erroneous assignments. The CEGs were clustered on $100 \%$ sequence identity and coverage using Python, and their genomic locations (chromosome or plasmid) were predicted using MOB-RECON [11]. 
109 Results

110

\section{Carbapenemase-encoding genes in bacterial genomes}

112 After RGI verification of annotated antibiotic resistance genes from PATRIC, we obtained nearly 1132 million ARGs from over 170,000 bacterial genomes (covering almost 11,000 species), from 114 which set we identified 9,892 carbapenemase-encoding genes in 7,964 bacterial genomes. The set 115 of 9,892 CEGs consisted of 203 unique protein sequences and 257 unique nucleotide sequences, 116 respectively. The former reflect the amino-acid based beta-lactam classifications system used by the BLDB [10] that allowed accurate assignment of each CEG to its protein name. Genes encoding KPC-2, OXA-23, OXA-66, and KPC-3 were detected over 1000 times in our dataset. The 7,964 genomes belonged to at least 77 bacterial species (18 lacked taxonomic assignment beyond the genus level, Supplemental File 4), from 33 genera, 18 families and 5 phyla.

121

122

123

124

125

126

127

128

129

130

131

132

133

134

135

136

137

138

139

140

141

142

143

144

145

146

\section{Multiple occurrences of CEGs within a single genome}

We found that while the majority $(6,191)$ of genomes only encoded a single CEG, 1,773 genomes carried multiple CEGs. Of the genomes carrying multiple CEGs, 189 harboured CEGs encoding the same protein, with OXA-23 encoding genes being most frequently present in multiple copies (41 genomes), followed by KPC- 3 encoding genes ( $\mathrm{n}=35)$, KPC- 2 encoding genes ( $\mathrm{n}=30)$, NDM- 1 encoding genes $(\mathrm{n}=12)$, and OXA-72 encoding genes $(\mathrm{n}=9)$. Interestingly, we found 38 genomes containing three or more genes encoding the same protein, and three genomes with five copies for the same protein, KPC-2 for Klebsiella michiganensis, and OXA-23 and OXA-72 for Acinetobacter baumannii. Moreover, we also found multiple gene copies encoding for the same protein in less common pathogens, such as three copies encoding KPC-3 in Serratia marcescens and Raoultella planticola.

Most of the genomes with multiple CEGs carried genes encoding different proteins ( $\mathrm{n}=1,666)$, which were of the same carbapenemase type for the majority of these genomes $(n=1,533)$. Combinations of OXA (oxacillinase) type CEGs were most common, especially $b l a_{\mathrm{OXA}-23}$ and $b l a_{\text {OXA-66 }}$ (803 genomes) and bla $a_{\text {OXA-23 }}$ and $b l a_{\text {OXA-82 }}(290$ genomes). However, 133 genomes contained CEGs encoding carbapenemases of two different types. The most frequent combination of genes encoding distinct carbapenemase types, i.e. $b l a_{\mathrm{NDM}-1}$ and $b l a_{\mathrm{OXA}-232}$ were found in 25 Klebsiella pneumoniae genomes and one Escherichia coli genome. Other common combinations were $b l a_{\mathrm{NDM}-1}$ and $b l a_{\mathrm{OXA}-94}$ in 11 Acinetobacter baumannii genomes, bla $_{\mathrm{KPC}-2}$ and bla $_{\mathrm{NDM}-1}$ in five Enterobacter cloacae, two Klebsiella pneumoniae, two Klebsiella michiganensis, and one Citrobacter freundii, and lastly bla $a_{\mathrm{NDM}-1}$ and bla $a_{\mathrm{OXA}-23}$ in 10 Acinetobacter baumannii genomes, one Acinetobacter radioresistens genome and one Acinetobacter sp. genome.

\section{Distribution of CEGs between species and environments}

Most CEGs were found in the genera Acinetobacter (3074 genomes : 4783 CEGs), Klebsiella (3267 : 3404), Escherichia (542: 559), Enterobacter (485: 502) and Pseudomonas (268: 281). Overall, CEGs were found most frequently present in the species Acinetobacter baumannii $(4,502$ 
147 CEGs), Klebsiella pneumoniae (3,285 CEGs), Escherichia coli (557 CEGs), Enterobacter cloacae 148 (313), Pseudomonas aeruginosa (245 CEGs), and also, albeit to less extent, in species less 149 commonly included in carbapenemase surveillance such as Serratia marcescens (66 CEGs), 150 Citrobacter freundii (59 CEGs), Klebsiella oxytoca (36), Klebsiella quasipneumoniae (27), 151 Klebsiella michiganensis (24 CEGs), and Proteus mirabilis (24 CEGs), and Acinetobacter 152 radioresistens (20 CEGs). Supplemental File 5 presents the numbers of genomes and CEGs. The 153 important CEG bla IMP-1 $_{1}$ was most often detected in Enterobacter hormaechei and Klebsiella 154 pneumoniae, but also in Acinetobacter baumannii, A. junii, A. pittii, A. nosocomialis, Enterobacter 155 cloacae, E. kobei, E. asburiae, Escherichia coli, Pseudomonas aeruginosa and Serratia 156 marcescens. bla $a_{\mathrm{OXA} 48}$ was predominantly found in K. pneumoniae, but also in Escherichia coli, 157 Enterobacter aerogenes, E. cloacae, E. hormaechei, E. kobei, Citrobacter freundii, C. koseri, 158 Shewanella sp. (on chromosome), Kluyvera ascorbata, Proteus mirabilis and Raoultella 159 ornithinolytica. bla $a_{\mathrm{NDM}-1,}$ bla $\mathrm{KPC}-2_{2}$ and bla $a_{\mathrm{KPC}-3}$ were most often identified in K. pneumoniae, 160 followed by E. coli and E. cloacae (Supplemental Files 6, 7 and 8).

161 Nucleotide sequence comparison of the 9,892 CEGs revealed that 1,591 CEG sequences were 162 present in only a single bacterial species, whereas the remaining 8,301 CEG sequences were found identically in multiple species. For example, the well-known CEGs $b l a_{N D M-1}, b l a_{K P C-2}, b l a_{I M P-1}$ and bla $_{O X A-48}$ in at least $38,27,12$ and 13 species, respectively.

165

166

167

168

169

170

171

172

173

174

175

176

177

178

179

Based on the isolation information for each genome we distinguished 11 different environments of CEGs, of which the human isolates represented by far the largest environment in this study $(8,099$ CEGs, $82 \%)$. CEGs such as bla $_{K P C-2}$, bla $_{\text {OXA-23 }}$ and bla $a_{\text {OXA-66 }}$ were dominant when considering all environments. However, when the human and miscellaneous (which will likely contain many human isolates) genomes were excluded from the dataset, dominant CEGs shifted, leading to high frequencies of $b l a_{\mathrm{NDM}-5}, b l a_{\mathrm{NDM}-9}$ and $b l a_{\mathrm{NDM}-1}$, mostly identified in bacteria isolated from chickens (109 genomes). The distribution of CEGs over the different species and environments is presented in Figure 1.

\section{Genomic location of CEGs}

We evaluated the genomic location of CEGs based on their genomic context and found that 4,137 (42\%) were predicted to be chromosomally located while 5,753 (58\%) were predicted to be plasmid-located. bla $a_{\mathrm{IMP} 1}$ was most often located on IncN or IncW plasmids, bla $a_{\mathrm{KPC}-2}$ on IncFII or IncU plasmids, $b l a_{\mathrm{KPC}-3}$ on IncFIA or IncFII plasmids, $b l a_{\mathrm{NDM}-1}$ on IncA/C plasmids, bla $a_{\mathrm{NDM}-5}$ on IncX3 plasmids and bla $_{\mathrm{OXA}-48}$ on Inc $\mathrm{L} / \mathrm{M}$ plasmids. 


\section{Discussion}

181

182

183

184

185

186

187

188

189

190

191

192

193

194

195

196

197

198

199

200

201

202

203

204

205

206

207

208

209

210

211

212

213

214

215

216

217

218

219

The identification of CEGs in a large number of genomes of a diversity of bacterial species showed that the distribution of these high-priority resistance genes extends far beyond the well-known clinically relevant carbapenem-resistant Acinetobacter baumannii, Pseudomonas aeruginosa and Enterobacteriaceae, which are known to be emerging causes for hospital acquired infections (https://www.who.int/infection-prevention/publications/guidelines-cre/en/).

A recent cross-border collaborative effort on whole genome sequencing of certain carbapenemase positive K. pneumoniae identified muli-country transmission clusters [12]. This analysis relied on 143 genome sequences, and underlined the benefits of data sharing. The integration of public data sources, such as the PATRIC genome repository with a backdrop of over 3,200 carbapenemase containing $K$. pneumoniae genomes, in addition to a substantive potential reservoir of mobile carbapenemase-encoding genes, may provide further context and epidemiological links for both strains as well as mobile elements outside the regularly surveilled species.

A considerable number of these genomes carried two or more different CEGs (Table 1). A consequence of co-expression of different types of carbapenemase genes, for example $b l a_{\mathrm{OXA}-48}$ and $b l a_{\mathrm{NDM}-1}$, together with other resistance genes that might be present on the plasmids, is a reduction of treatment options [13]. In this study, only carbapenemase-encoding genes were included in the analyses, but their co-occurrence with antibiotic resistance genes from completely different classes, such as aminoglycosides, tetracyclines, et cetera, may be of interest as well to evaluate the emergence of multidrug resistant pathogens.

We found that identical CEGs are frequently shared between bacterial species and -as a consequence- can be encountered in a large number of environments (Figure 1). Horizontal gene transfer is an important evolutionary force for adaptation, and mobile genetic elements are wellknown vectors for gene exchange, especially for antibiotic resistance genes [14]. The high percentage $(58 \%)$ of predicted plasmid-located CEGs in the genomes indeed shows that a large proportion of CEGs have an enhanced capacity for horizontal gene transfer. Previous genomic epidemiology studies may not have fully appreciated the widespread character of CEG dissemination [15], and possible co-mobility with other resistance determinants.

The database approach identified human isolates as the largest reservoir of CEGs. This however does not necessarily mean that the human environment is the dominant reservoir for carbapenemresistance genes, but rather may reflect the frequent sampling of humans. In addition, it is likely that many more CEG-environment combinations exist as this analysis is limited to the biased isolates present in the database. Indeed, most non-human environments harbor multiple CEG variants (Table 2), despite the fact that they contain relatively few isolates .

PeerJ reviewing PDF | (2020:06:50324:1:2:NEW 11 Jan 2021) 


\section{Conclusions}

222 Large genome repositories are treasure troves for comparative analyses in different fields of study, 223 even though these databases do not represent surveillance data and therefore contain bacterial 224 isolates at other frequencies compared to their isolation sources, nor are they free of annotation 225 errors. Still, owing to their vast sizes, genome sequence collections allow for an alternative view 226 on the distribution of resistance genes and exchange potential between bacteria and environments. 227 Carbapenemase-encoding genes are distributed over a large number of bacterial species, with some 228 CEGs, such as $b l a_{N D M-1}$, present in at least 42 different species, isolated from at least seven distinct 229 non-human environments. These numbers show the breadth of reservoirs and the vast potential for 230 resistance gene exchange and transmission. Data sharing and the use of open repositories for 231 surveillance purposes would therefore benefit evaluations of health risks for resistant infections. 


\section{References}

234

235

236

237

238

239

240

241

242

243

244

245

246

247

248

249

250

251

252

253

254

255

256

257

258

259

260

261

262

263

264

265

266

267

268

269

270

271

272

273

274

275

276

1. Wattam, A.R., J.J. Davis, R. Assaf, S. Boisvert, T. Brettin, C. Bun, N. Conrad, E.M. Dietrich, T. Disz, J.L. Gabbard, S. Gerdes, C.S. Henry, R.W. Kenyon, D. Machi, C. Mao, E.K. Nordberg, G.J. Olsen, D.E. Murphy-Olson, R. Olson, R. Overbeek, B. Parrello, G.D. Pusch, M. Shukla, V. Vonstein, A. Warren, F. Xia, H. Yoo, and R.L. Stevens, Improvements to PATRIC, the all-bacterial Bioinformatics Database and Analysis Resource Center. Nucleic Acids Res, 2017. 45(D1): p. D535-D542.

2. Mao, C., D. Abraham, A.R. Wattam, M.J. Wilson, M. Shukla, H.S. Yoo, and B.W. Sobral, Curation, integration and visualization of bacterial virulence factors in PATRIC. Bioinformatics, 2015. 31(2): p. 252-8.

3. Karp, P.D., S.M. Paley, M. Krummenacker, M. Latendresse, J.M. Dale, T.J. Lee, P. Kaipa, F. Gilham, A. Spaulding, L. Popescu, T. Altman, I. Paulsen, I.M. Keseler, and R. Caspi, Pathway Tools version 13.0: integrated software for pathway/genome informatics and systems biology. Brief Bioinform, 2010. 11(1): p. 40-79.

4. Antonopoulos, D.A., R. Assaf, R.K. Aziz, T. Brettin, C. Bun, N. Conrad, J.J. Davis, E.M. Dietrich, T. Disz, S. Gerdes, R.W. Kenyon, D. Machi, C. Mao, D.E. Murphy-Olson, E.K. Nordberg, G.J. Olsen, R. Olson, R. Overbeek, B. Parrello, G.D. Pusch, J. Santerre, M. Shukla, R.L. Stevens, M. VanOeffelen, V. Vonstein, A.S. Warren, A.R. Wattam, F. Xia, and $\mathrm{H}$. Yoo, PATRIC as a unique resource for studying antimicrobial resistance. Brief Bioinform, 2019. 20(4): p. 1094-1102.

5. Rello, J., V. Kalwaje Eshwara, L. Lagunes, J. Alves, R.G. Wunderink, A. ConwayMorris, J.N. Rojas, E. Alp, and Z. Zhang, A global priority list of the TOp TEn resistant Microorganisms (TOTEM) study at intensive care: a prioritization exercise based on multi-criteria decision analysis. Eur J Clin Microbiol Infect Dis, 2019. 38(2): p. 319-323.

6. Kock, R., I. Daniels-Haardt, K. Becker, A. Mellmann, A.W. Friedrich, D. Mevius, S. Schwarz, and A. Jurke, Carbapenem-resistant Enterobacteriaceae in wildlife, foodproducing, and companion animals: a systematic review. Clin Microbiol Infect, 2018. 24(12): p. 1241-1250.

7. Brolund, A., N. Lagerqvist, S. Byfors, M.J. Struelens, D.L. Monnet, B. Albiger, A. Kohlenberg, and E.-N.C.S.G. European Antimicrobial Resistance Genes Surveillance Network, Worsening epidemiological situation of carbapenemase-producing Enterobacteriaceae in Europe, assessment by national experts from 37 countries, July 2018. Euro Surveill, 2019. 24(9).

8. Woodford, N., D.W. Wareham, B. Guerra, and C. Teale, Carbapenemase-producing Enterobacteriaceae and non-Enterobacteriaceae from animals and the environment: an emerging public health risk of our own making? J Antimicrob Chemother, 2014. 69(2): p. 287-91.

9. Jia, B., A.R. Raphenya, B. Alcock, N. Waglechner, P. Guo, K.K. Tsang, B.A. Lago, B.M. Dave, S. Pereira, A.N. Sharma, S. Doshi, M. Courtot, R. Lo, L.E. Williams, J.G. Frye, T. Elsayegh, D. Sardar, E.L. Westman, A.C. Pawlowski, T.A. Johnson, F.S. Brinkman, G.D. Wright, and A.G. McArthur, CARD 2017: expansion and model-centric curation of the comprehensive antibiotic resistance database. Nucleic Acids Res, 2017. 45(D1): $\mathrm{p}$. D566-D573.

Peer] reviewing PDF | (2020:06:50324:1:2:NEW 11 Jan 2021) 
277 10. Naas, T., S. Oueslati, R.A. Bonnin, M.L. Dabos, A. Zavala, L. Dortet, P. Retailleau, and

278

279

280

281

282

283

284

285

286

287

288

289

290

291

292

293

294

295

296

297

298

299 B.I. Iorga, Beta-lactamase database (BLDB) - structure and function. J Enzyme Inhib Med Chem, 2017. 32(1): p. 917-919.

11. Robertson, J. and J.H.E. Nash, MOB-suite: software tools for clustering, reconstruction and typing of plasmids from draft assemblies. Microb Genom, 2018. 4(8).

12. Ludden, C., F. Lotsch, E. Alm, N. Kumar, K. Johansson, B. Albiger, T.D. Huang, O. Denis, A.M. Hammerum, H. Hasman, J. Jalava, K. Raisanen, L. Dortet, A.B. Jousset, S. Gatermann, S. Haller, M. Cormican, W. Brennan, M. Del Grosso, M. Monaco, L. Schouls, O. Samuelsen, M. Pirs, T. Cerar, J. Oteo-Iglesias, M. Perez-Vazquez, K. Sjostrom, P. Edquist, K.L. Hopkins, M.J. Struelens, D. Palm, D.L. Monnet, and A. Kohlenberg, Cross-border spread of bla NDM-1- and bla OXA-48-positive Klebsiella pneumoniae: a European collaborative analysis of whole genome sequencing and epidemiological data, 2014 to 2019. Euro Surveill, 2020. 25(20).

13. Gona, F., D. Bongiorno, A. Aprile, E. Corazza, B. Pasqua, M.G. Scuderi, M. Chiacchiaretta, D.M. Cirillo, S. Stefani, and M.L. Mezzatesta, Emergence of two novel sequence types (3366 and 3367) NDM-1- and OXA-48-co-producing K. pneumoniae in Italy. Eur J Clin Microbiol Infect Dis, 2019. 38(9): p. 1687-1691.

14. Ding, B., Z. Shen, F. Hu, M. Ye, X. Xu, Q. Guo, and M. Wang, In vivo Acquisition of Carbapenemase Gene blaKPC-2 in Multiple Species of Enterobacteriaceae through Horizontal Transfer of Insertion Sequence or Plasmid. Front Microbiol, 2016. 7: p. 1651.

15. Wilson, H. and M.E. Torok, Extended-spectrum beta-lactamase-producing and carbapenemase-producing Enterobacteriaceae. Microb Genom, 2018. 4(7). 


\section{Figure 1}

Distribution of the 9,892 carbapenemase-encoding genes in bacterial species and nonhuman reservoirs.

Horizontal lines represent reservoirs (A) or bacterial species (B) that collectively harbor different CEGs. Vertical lines represent CEGs that occur in many different bacterial species or reservoirs: four genes have been highlighted. The figure was simplified by removing the rare CEGs, and the full data is available in Supplemental files 6, 7 and 8. 


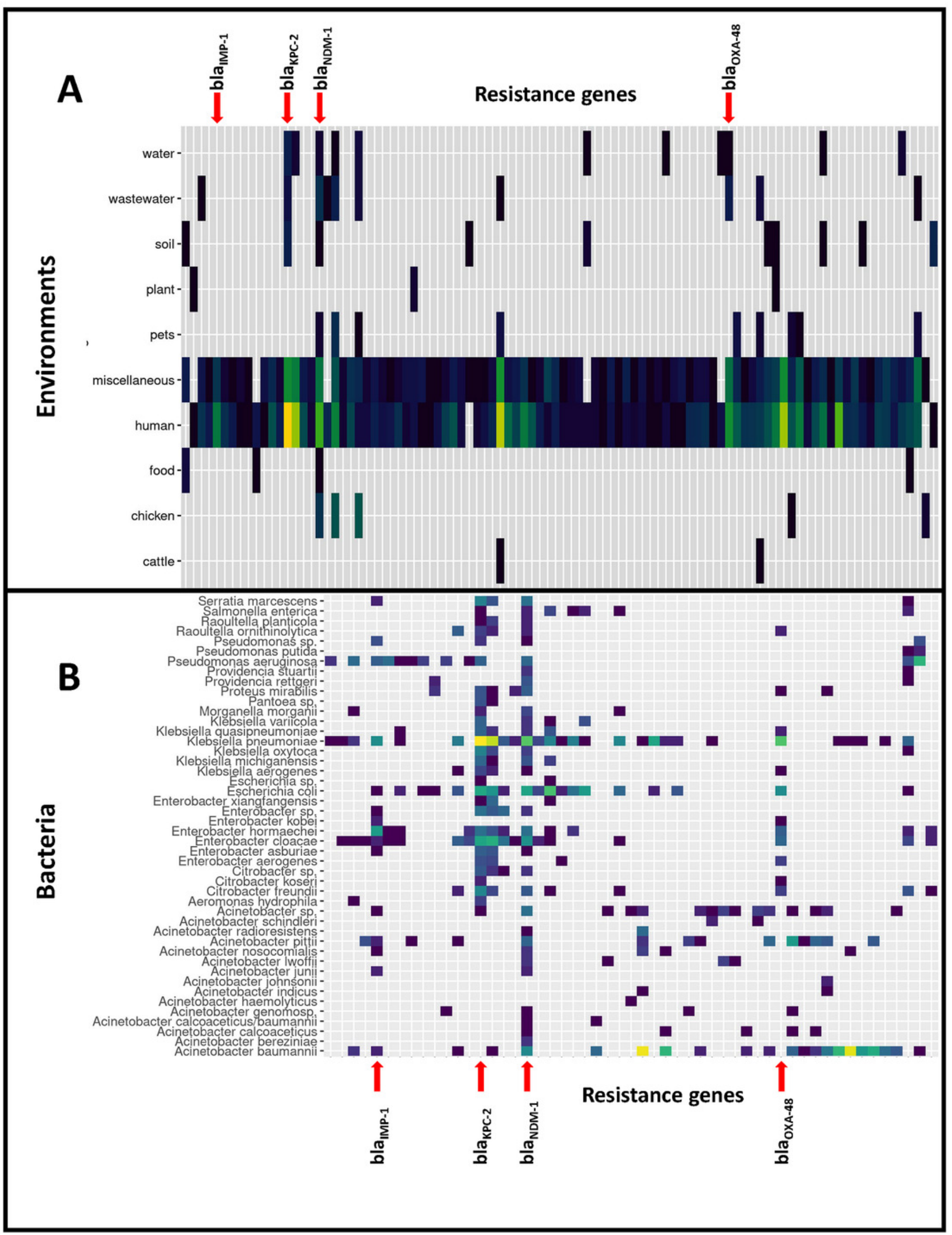




\section{Table $\mathbf{1}$ (on next page)}

Distribution of carbapenemase-encoding genes (CEG) in bacterial genomes.

The 9,892 CEGs occur in 7,884 bacterial genomes. The majority of genes $(6,191)$ is present in a single copy in its genome, but 3,701 genes reside in a genome with additional carbapenemase-encoding genes 
1

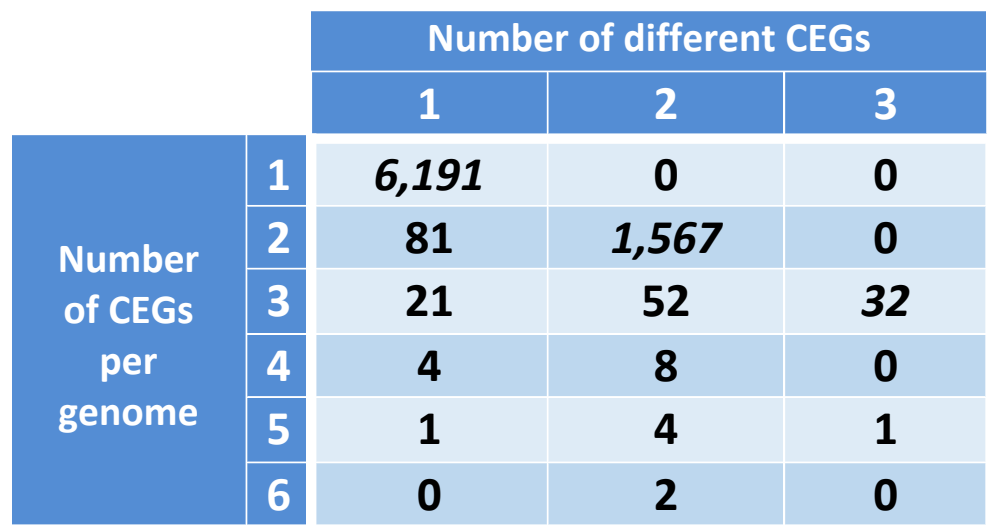

2
3

3 
Table 2 (on next page)

Diversity of CEGs in the 11 reservoirs distinguished in the genome database 


\begin{tabular}{|c|c|c|c|c|}
\hline Environment & $\begin{array}{l}\text { \# Distinct } \\
\text { CEGs }\end{array}$ & CEGs & \# Species & Species* \\
\hline Pig & 1 & NDM-1 & 1 & A. baumannii \\
\hline Cattle & 3 & OXA-146,OXA-23,OXA-58 & 1 & A. indicus \\
\hline Plant & 3 & CGB-1,OXA-120,OXA-65 & 2 & A. baumannii, C. indologenes, \\
\hline Chicken & 5 & $\begin{array}{l}\text { NDM-1,NDM-5,NDM-9,OXA- } \\
68, \text { VIM-48 }\end{array}$ & 5 & A. baumannii, E. coli, K. pneumoniae, S. enterica, P. putida \\
\hline Food & 5 & $\begin{array}{l}\text { Bcll-1,IMP-27,NDM-1,OXA- } \\
\text { 497,VIM-1 }\end{array}$ & 6 & $\begin{array}{l}\text { A. baumanni, B. cereus, B. thuringensis, E. coli, V. } \\
\text { parahaemolyticus, S. enterica }\end{array}$ \\
\hline Pets & 9 & $\begin{array}{l}\text { NDM-1,NDM-5,NDM-9,OXA- } \\
\text { 23,OXA-500,OXA-58,OXA- } \\
\text { 68,OXA-69,VIM-2 }\end{array}$ & 6 & $\begin{array}{l}\text { A. baumannii, A. gandensis, A. pittii, A. radioresistens, E. } \\
\text { coli, P. aeruginosa }\end{array}$ \\
\hline Soil & 10 & $\begin{array}{l}\text { Bcll-1,KPC-2,NDM-1,OXA- } \\
\text { 213,OXA-273,OXA-64,OXA- } \\
\text { 65,OXA-72,OXA-91,VIM-5 }\end{array}$ & 10 & $\begin{array}{l}\text { A. baumannii, A. calcoaceticus, Acinetobacter sp., } B . \\
\text { cereus, B. subtilis, C. braakii, C. freundii, Erythrobacter sp., } \\
\text { P. guariconensis, P. plecoglossicida }\end{array}$ \\
\hline Wastewater & 11 & $\begin{array}{l}\text { GES-5, KPC-2,NDM-1,NDM- } \\
\text { 4,NDM-5,NDM-9,OXA- } \\
\text { 23,OXA-48,OXA-58,SHV- } \\
\text { 38,VIM-2 }\end{array}$ & 11 & $\begin{array}{l}\text { A. johnsonii, A. junii, Acinetobacter sp., A. hydrophila, C. } \\
\text { freundii, E. cloacae, E. kobei, E. coli, Escherichia sp., K. } \\
\text { pneumoniae, Pseudomonas sp. }\end{array}$ \\
\hline Water & 13 & $\begin{array}{l}\text { KPC-2,KPC-3,NDM-1,NDM- } \\
\text { 5,NDM-9,OXA-269,OXA- } \\
\text { 273,OXA-280,OXA-360,OXA- } \\
\text { 444,OXA-48,OXA-72,SPM-1 }\end{array}$ & 14 & $\begin{array}{l}\text { A. calcoaceticus, A. johnsonii, A. pittii, A. schindleri, E. } \\
\text { cloacae, E. coli, G. pentaromativorans, K. pneumoniae, K. } \\
\begin{array}{llll}\text { quasipneumoniae, } \quad \text { K. } \quad \text { variicola, } & \text { P. aeruginosa, } \\
\text { Pseudomonas sp., Ralstonia sp., V. cholerae }\end{array}\end{array}$ \\
\hline Miscellaneous & 109 & $* *$ & 48 & $* * *$ \\
\hline Human & 175 & $* *$ & 73 & $* * *$ \\
\hline
\end{tabular}

3 *) for the full names, please refer to Supplemental File 4

$\left.4{ }^{* *}\right)$ the list of genes is in the Supplemental File 6

$5 * * *$ ) the list of species is in the Supplemental File 7 